\title{
Traduire
}

Une eutre perspective sur $r$ tatadciction

Revue française de la traduction

$224 \mid 2011$

Des outils et des nuages

\section{Rétrospective $\mathrm{n}^{\circ} 17$}

\section{Maurice Voituriez}

\section{(2) OpenEdition \\ Journals}

Édition électronique

URL : http://journals.openedition.org/traduire/239

DOI : 10.4000/traduire.239

ISSN : 2272-9992

Éditeur

Société française des traducteurs

Édition imprimée

Date de publication : 1 juin 2011

Pagination : 143-145

ISSN : 0395-773X

\section{Référence électronique}

Maurice Voituriez, «Rétrospective n 17 », Traduire [En ligne], 224 | 2011, mis en ligne le 03 février 2014, consulté le 15 septembre 2020. URL : http://journals.openedition.org/traduire/239 


\section{Rétrospective $n^{\circ} 17$}

\section{Maurice Voituriez}

En 1988 la SFT avait encore un stand à EXPOLANGUES et la FIT (Fédération internationale des traducteurs) bénéficiait de son hospitalité. La présence sur un stand n'était pas alors le seul événement mais constituait l'occasion d'une rencontre privilégiée avec nos confrères britanniques de I'ITI (Institute of Translation and interpreting) et de journées de travail consacrées à la "traduction face aux nouveaux médias".

À cette époque le traitement de texte existe déjà mais tout le monde ne dispose pas encore d'un ordinateur dans notre profession. II s'en faut ! Nous-mêmes avions depuis 1975 un appareil dénommé AMSTRAD PCW 8256 ne comportant pas de disque dur mais des disquettes de 3". Pas de comptage des mots ni de correcteur orthographique. Dans les années 80 le disque dur arrivait, en même temps que des logiciels performants. En revanche, la télévision était déjà omniprésente avec les conséquences suivantes : internationalisation de la publicité, invasion des programmes transmis par satellites. Pour y faire face, une nouvelle race de professionnels commence à émerger, celle des communicateurs. À la fois traducteurs, rédacteurs, adaptateurs, ils doivent tout savoir faire.

Le traducteur audiovisuel est un professionnel hybride. Ce peut être, en plus, un interprète, lors d'émissions en direct. II peut se limiter au sous-titrage. Dans les deux cas il y a une contrainte de temps. Cela n'a pas dû changer après deux décennies! Un autre aspect du travail est l'adaptation de messages et de slogans publicitaires d'une langue à l'autre.

Un exemple cité par Françoise Guilliard pour I'AÉROSPATIALE :

\section{NOTRE EXPÉRIENCE, NOUS NE L'AVONS PAS VOLÉE} WE HAVE EARNED OUR WINGS

Toujours Porte de Versailles mais cette fois-ci dans le cadre du Salon du Livre, un colloque se tenait sur le thème "Qui traduit les livres ? ". Huguette Brusick, présidente de la SFT à l'époque, animait les débats. Un seul éditeur était présent et participait. II s'agissait du très dynamique "Actes Sud " ici représenté par Bertrand Py et publiant en effet bon nombre de traductions. 
II mentionne néanmoins le fait que, dans des perspectives commerciales comparables, un livre traduit coûte plus cher qu'un livre directement écrit en français, à cause de l'à-valoir payé au traducteur et du délai de récupération de la mise de fonds plus long (puisqu'il faut compter le temps de réalisation du travail). C'est bien exact mais on pourrait objecter que l'éditeur assume moins de risques, sachant quel accueil le livre a rencontré dans sa langue d'origine (et éventuellement en traduction dans d'autres langues) et ayant recueilli les opinions et témoignages de lecteurs bilingues recrutés à cette fin. Quant au traducteur, il touchera rarement plus que l'à-valoir et n'accèdera à son pourcentage de droit d'auteur que si le livre atteint un gros tirage. Dans le domaine technique c'est encore plus rarement le cas. Personnellement, pour la réalisation d'un dictionnaire relatif à la parachimie, nous avons perçu des droits pendant vingt ans. Ils étaient importants les premières années, très faibles à la fin, ce qui n'a rien d'anormal. Vingt ans, ce n'est pas rien!

Commenter un article intitulé "LE CHINOIS, langue de l'avenir ? ", c'est un peu enfoncer une porte ouverte. "Dans vingt ans - écrit P. Dentadour - plus aucun cadre chinois ne parlera le français. L'anglais, le japonais, l'allemand et même l'italien sont en train de prendre le pas sur la langue de Molière... et de Bernard Tapie."

Par parenthèse Molière et Bernard Tapie forment ici un étrange duo!

Rappelons que la France du Général de Gaulle fut le premier pays de l'Europe occidentale à ouvrir en 1962 des relations diplomatiques avec la Chine. Cette initiative fut chez nous critiquée à l'époque et c'est soixante ans après qu'on en mesure la pertinence. "Le chinois : apprendre pour entreprendre " : ce titre de l'exposé de M. Dentadour, ancien directeur du Crédit Lyonnais de Singapour, reste aujourd'hui bien actuel. Donnons-lui à nouveau la parole :

Tout praticien des langues étrangères sait que son efficacité est directement proportionnelle à l'amour qu'il porte à la culture véhiculée par ces langues. Et c'est peut-être l'ingrédient qui manque dans la démarche de nos commerciaux, trop pressés d'adhérer au club du franglais, trop conditionnés par le facile sabir atlantique pour s'atteler à l'étude longue, mais éminemment profitable, d'une langue différente et riche d'une autre pensée, qui, demain, donnera accès à de vastes marchés nouveaux!

Tout cela est très vrai. Avec cette réserve que l'anglais n'est pas le franglais et qu'on peut porter une véritable dilection à la culture et à la littérature britannique, ce qui n'exclut, du reste, pas l'amour de la langue allemande.

À notre regret nous ne connaissons pas le chinois mais nous avons une jeune Chinoise dans notre famille!

Retournons en Europe avec un séjour à Berlin-Est en 1988. Invités par l'association des traducteurs de la RDA, nous y avons passé une semaine inoubliable. 
Relisant notre article dans Traduire, nous avons retenu le passage suivant :

Certes le Mur (construit en 1961) est toujours là mais quelques gestes humanitaires ou touristiques en atténuent parfois la rigueur. On dit que des négociations ont lieu. Si c'est vrai, puissent-elles aboutir! Imaginons Paris coupé en deux, la rive gauche séparée de la rive droite et tous les ponts (sauf un seul, équivalent du Checkpoint Charlie) barrés par un mur et des barbelés. Telle est la situation que vivent depuis près de trente ans les Berlinois.

Ce que nous espérions s'est produit. Le Mur est tombé en 1989. Nous avons cependant gardé un bon souvenir de Berlin et de Potsdam, malgré le régime existant à l'époque. L'excellent accueil de nos confrères berlinois y a fortement contribué.

Quand nous écrivions régulièrement dans notre revue, la rédactrice en chef était à l'époque notre amie Denise Baccara. Si elle lit ces lignes, qu'elle sache que je garde de notre collaboration un fidèle et excellent souvenir !

maurice.voituriez@dbmail.com 